\title{
Gravitational self-force corrections to tidal invariants for spinning particles on circular orbits in a Schwarzschild spacetime
}

\author{
Donato Bini ${ }^{1}$ and Andrea Geralico ${ }^{1}$ \\ ${ }^{1}$ Istituto per le Applicazioni del Calcolo "M. Picone," CNR, I-00185 Rome, Italy
}

(Dated: June 12, 2018)

\begin{abstract}
We compute gravitational self-force corrections to tidal invariants for spinning particles moving along circular orbits in a Schwarzschild spacetime. In particular, we consider the square and the cube of the gravitoelectric quadrupolar tidal tensor and the square of the gravitomagnetic quadrupolar tidal tensor. Our results are accurate to first-order in spin and through the 9.5 post-Newtonian order. We also compute the associated electric-type and magnetic-type eigenvalues.
\end{abstract}

\section{INTRODUCTION}

The recent detections of gravitational wave signals by the Ligo and Virgo collaborations [1-4] have strengthen more and more our motivations and efforts to improve the theoretical understanding of the general relativistic dynamics of binary systems made of compact objects endowed with spin, quadrupole, and even higher multipolar structure. In this context tidal interactions are expected to play an important role. For instance, gravitational waves emitted during the coalescence of binary neutron stars are expected to contain an imprint of the tidal interaction between the two bodies [ $5-12]$.

In a zeroth-order approximation level, the mass of one body can be considered much smaller than that of the other, in such a way that backreaction effects can also be neglected and the two-body dynamics reduces to the motion of an extended body in a given gravitational field due to the body of higher mass. The smaller body thus undergoes tidal deformations which can be studied, e.g., by constructing a body-fixed frame adapted to the timelike geodesic representative of its motion under the action of a tidal potential in terms of Fermi-type coordinates [13]. Alternatively, one can use an effective field theory approach to dynamical tides consisting in modifying the point mass action with the addition of certain nonminimal couplings involving integrals of tidal invariant quantities performed along the body's world line [14 19]. Such invariants are constructed through the electric-type and magnetic-type tidal tensors given by the tensorial contraction of the spacetime Riemann tensor and its dual, respectively, with the tensor product of the body's fourvelocity with itself. Actually, such an effective action description of tidal effects is a general framework holding in perturbation theory too, where the backreaction of the body on the background geometry is taken into account, as well as in post-Newtonian theory [20].

The mathematical modeling of the two-body tidal problem in complete generality is much complicated due to the impossibility of exploring through analytical methods the strong-field regime (occurring typically at the end of the coalescence process, when the merging phase starts) and the associated effects, so that one has mainly to rely on numerical analyses. Recent years have witnessed a very useful strategy to investigate strong-field effects which combines, in a synergic way, information coming from various approximation methods, namely: the post-Newtonian (PN) formalism (see, e.g., Ref. 21] and references therein), the post-Minkowskian (PM) one 22 24], the gravitational self-force (GSF) formalism (see, e.g., Ref. 25] and references therein), full numerical relativity simulations $26-28$, and the effective one-body (EOB) formalism [29 32]. In particular, the EOB approach has proven to be a key framework where one can combine efficiently information coming from all the other approximation schemes, with the advantage that EOBbased simulations are very fast (and accurate), and thousands of EOB templates have been built over the last years [33, 34]. At the moment, the inclusion in the EOB Hamiltonian of tidal interaction terms is still under study. For instance, Ref. [35] considers finite size effects on the orbital dynamics of a compact body like a neutron star by modifying the (effective) point-particle action adding quadrupolar degrees of freedom, which in turn allow to define "dynamical tides" all along the inspiral process.

In the present paper, we extend previous works on tidal invariants along circular orbits in a Schwarzschild spacetime obtained for spinless particles [19, 20, 36 40] to the case of spinning bodies to linear order in spin in the extreme-mass-ratio limit. This work belongs to a project studying gravitational self-force corrections due to the multipolar structure of the perturbing body, already started with the computation of the Detweiler's redshift invariant [41]. As standard, the dynamics of the small spinning body is described according to the MathissonPapapetrou-Dixon (MPD) model [42 44]. The orbit is assumed to be circular and equatorial, the spin vector being orthogonal to the plane of motion.

We expect that the present work will give additional (high-PN order, first-GSF order) information to be used to improve both modeling and simulations of the twobody dynamics in the extreme mass ratio and small spin limits.

The masses of the gravitationally interacting two bodies are denoted by $m_{1}$ and $m_{2}$, with the convention that $m_{1} \leq m_{2}$. We then define

$$
M \equiv m_{1}+m_{2}, \quad \mu \equiv \frac{m_{1} m_{2}}{M}, \quad \nu \equiv \frac{\mu}{M},
$$

as the total mass, reduced mass and symmetric mass ratio, respectively. We shall also use the other dimension- 
less mass ratios

$$
q \equiv \frac{m_{1}}{m_{2}}, \quad X_{1} \equiv \frac{m_{1}}{M}, \quad X_{2} \equiv \frac{m_{2}}{M}=1-X_{1}
$$

such that

$$
\nu=\frac{q}{(1+q)^{2}}, \quad X_{1}=\frac{q}{1+q}, \quad X_{2}=\frac{1}{1+q} .
$$

In the small mass-ratio limit $q \ll 1$ we have $\nu \simeq X_{1} \simeq$ $q \ll 1$ and $X_{2}=1-q+O\left(q^{2}\right)$.

In the following we will set $G=c=1$ and use the signature +2 .

\section{SPINNING PARTICLE MOTION IN A PERTURBED SCHWARZSCHILD SPACETIME}

Let us consider a spinning particle with mass $m_{1}$ moving in a perturbed Schwarzschild spacetime with line element

$$
d s^{2}=\left(\bar{g}_{\alpha \beta}+h_{\alpha \beta}\right) d x^{\alpha} d x^{\beta},
$$

where

$$
\bar{g}_{\alpha \beta} d x^{\alpha} d x^{\beta}=-f d t^{2}+f^{-1} d r^{2}+r^{2}\left(d \theta^{2}+\sin ^{2} \theta d \phi^{2}\right),
$$

with $f=1-2 m_{2} / r$, and $h_{\alpha \beta}=O(q)$. The motion is governed by the MPD equations [42 44], which read

$$
\begin{aligned}
m_{1} \frac{\mathrm{D} U^{\mu}}{d \tau} & =-\frac{1}{2} R_{\nu \alpha \beta}^{\mu} U^{\nu} S^{\alpha \beta}, \\
\frac{\mathrm{D} S^{\mu \nu}}{d \tau} & =O(2),
\end{aligned}
$$

to first-order in spin. Here we recall that $U^{\mu} \equiv d z^{\mu} / d \tau$ is the timelike unit tangent vector to the world line representative of the body's motion (with parametric equations $x^{\mu}=z^{\mu}(\tau)$ ) used to make the multipole reduction, parametrized by the proper time $\tau$, and the total 4-momentum of the particle is aligned with $U$, i.e., $P=m_{1} U+O(2)$. The (antisymmetric) spin tensor $S^{\mu \nu}$ is taken to satisfy the Tulczyjew-Dixon conditions [44, 45]

$$
S^{\mu \nu} P_{\nu}=m_{1} S^{\mu \nu} U_{\nu}=0
$$

The associated spin vector obtained by spatial duality

$$
S(U)^{\gamma}=\frac{1}{2} U_{\sigma} \eta^{\sigma \gamma \alpha \beta} S_{\alpha \beta}, \quad S^{\gamma} U_{\gamma}=0
$$

is parallel-propagated along $U, \nabla_{U} S^{\gamma}=0$. Both the particle's mass $m_{1}$ and the signed magnitude $s$ of the spin vector

$$
s^{2}=S(U)^{\beta} S(U)_{\beta}=\frac{1}{2} S_{\mu \nu} S^{\mu \nu},
$$

are constant along the path.

Let us assume that the perturbed metric admits the Killing vector $k=\partial_{t}+\Omega \partial_{\phi}$ and that the body's orbit is aligned with $k$ with spin vector directed orthogonal to the equatorial plane, i.e., it moves along a circular orbit $U=\Gamma k$ with

$$
-\Gamma^{-2}=k \cdot k=-f+\Omega^{2} r^{2}+h_{k k},
$$

where $h_{k k}=h_{\alpha \beta} k^{\mu} k^{\nu}$, and

$$
S=-s e_{\hat{\theta}}, \quad e_{\hat{\theta}}=\frac{1}{r}\left(1-\frac{1}{2 r^{2}} h_{\theta \theta}\right) \partial_{\theta} .
$$

The spin magnitude $s$ has a positive (negative) sign if the associated orbital angular momentum is parallel (respectively, antiparallel) to $e_{\hat{z}}=-e_{\hat{\theta}}$. It is also useful to introduce the associated dimensionless spin parameter $\hat{s} \equiv s /\left(m_{1} m_{2}\right)$.

The equations of motion (2.3) can then be cast in the form

$$
\begin{aligned}
m_{1} \Gamma \nabla_{k} k^{\mu} & =-\frac{1}{2}\left(\nabla_{\mu \beta} k_{\alpha}\right) S^{\alpha \beta} \\
& =-\frac{1}{2}\left(\nabla_{\mu} K_{\beta \alpha}\right) S^{\alpha \beta},
\end{aligned}
$$

where the (antisymmetric) tensor $K_{\alpha \beta}$ is given by

$$
K_{\alpha \beta}=\nabla_{\alpha} k_{\beta}=\partial_{[\alpha} k_{\beta]},
$$

and imply the following solution for $\Omega$

$$
m_{2} \Omega=u^{3 / 2}\left[1-\frac{3}{2} \hat{s} u^{3 / 2}+q\left(\tilde{\Omega}_{1}+\hat{s} \tilde{\Omega}_{1 \hat{s}}\right)\right] .
$$

Here

$$
\tilde{\Omega}_{1}=-\frac{m_{2}}{4 u^{2}} \partial_{r} h_{k k}^{(0)},
$$

and the spin correction

$$
\tilde{\Omega}_{1 \hat{s}}=\tilde{\Omega}_{1 \hat{s}}^{(h)}+\tilde{\Omega}_{1 \hat{s}}^{(\partial h)}+\tilde{\Omega}_{1 \hat{s}}^{\left(\partial^{2} h\right)},
$$

with 


$$
\begin{aligned}
\tilde{\Omega}_{1 \hat{s}}^{(h)}= & -\frac{u^{3 / 2}}{4(1-2 u)^{2}} h_{k k}^{(0)}+\frac{(5-12 u) u^{3 / 2}}{4} h_{r r}^{(0)}-\frac{u^{2}(3-4 u)(1-3 u)}{2 m_{2}(1-2 u)^{2}} h_{t \phi}^{(0)}-\frac{(1-3 u)\left(2-5 u+4 u^{2}\right) u^{5 / 2}}{4 m_{2}^{2}(1-2 u)^{2}} h_{\phi \phi}^{(0)}, \\
\tilde{\Omega}_{1 \hat{s}}^{(\partial h)}= & \frac{m_{2} u^{1 / 2}}{4(1-2 u)} \partial_{r} h_{k k}^{(0)}-\frac{m_{2}}{4 u^{2}} \partial_{r} h_{k k}^{(1)} \\
& +\frac{1}{4}(1-3 u)\left[-\partial_{\phi} h_{r k}^{(0)}+m_{2}(1-2 u) u^{-1 / 2} \partial_{r} h_{r r}^{(0)}+\frac{1}{(1-2 u)} \partial_{r} h_{t \phi}^{(0)}+\frac{(2-3 u) u^{3 / 2}}{m_{2}(1-2 u)} \partial_{r} h_{\phi \phi}^{(0)}\right], \\
\tilde{\Omega}_{1 \hat{s}}^{\left(\partial^{2} h\right)}= & -\frac{m_{2}^{2} u^{-3 / 2}}{4} \partial_{r r} h_{k k}^{(0)}-\frac{m_{2}}{4 u}(1-3 u)\left[\partial_{r r} h_{\phi k}^{(0)}-\partial_{r \phi} h_{r k}^{(0)}\right],
\end{aligned}
$$

where the metric components $h_{\alpha \beta}=h_{\alpha \beta}^{(0)}+\hat{s} h_{\alpha \beta}^{(1)}$ and their derivatives are evaluated at $r=m_{2} / u$.

It is useful to introduce the dimensionless frequency parameter $y=\left(m_{2} \Omega\right)^{2 / 3}$,

$$
y=u-\hat{s} u^{5 / 2}+q \mathcal{F}(u),
$$

to first order in $\hat{s}$, where $\mathcal{F}(u)=\mathcal{F}_{0}(u)+\hat{s} \mathcal{F}_{\hat{s}}(u)$. A direct calculation shows

$$
\begin{aligned}
& \mathcal{F}_{0}(u)=\frac{2}{3} u \tilde{\Omega}_{1}(u), \\
& \mathcal{F}_{\hat{s}}(u)=\frac{1}{3} u^{5 / 2} \tilde{\Omega}_{1}(u)+\frac{2}{3} u \tilde{\Omega}_{1 \hat{s}}(u) .
\end{aligned}
$$

This relation can be inverted to give

$$
u=y\left(1+\hat{s} y^{3 / 2}\right)+q\left[f_{0}(y)+\hat{s} f_{\hat{s}}(y)\right],
$$

to first order in $\hat{s}$, where

$$
\begin{aligned}
f_{0}(y) & =\frac{m_{2}}{6 y} \partial_{r} h_{k k}^{(0)}, \\
f_{\hat{s}}(y) & =\frac{y}{3}\left[\frac{m_{2}}{\sqrt{y}} \partial_{r} h_{k k}^{(0)}-2 \tilde{\Omega}_{1 \hat{s}}\right] .
\end{aligned}
$$

Finally, the following quantity is a constant of motion associated with the Killing vector $k$

$$
\begin{aligned}
E-\Omega J & =-m_{1} k_{\alpha} U^{\alpha}+\frac{1}{2} S^{\alpha \beta} \nabla_{\beta} k_{\alpha} \\
& =\frac{m_{1}}{\Gamma}+\Gamma K^{* \sigma \gamma} k_{\sigma} S_{\gamma} \\
& =m_{1} \Gamma^{-1}-m_{1} \hat{s} m_{2} b,
\end{aligned}
$$

so that

$$
\hat{E}-m_{2} \Omega \hat{J}=z_{1}-\hat{s} m_{2} b
$$

with $\hat{E}=E / m_{1}, \hat{J}=J /\left(m_{1} m_{2}\right)$ and $z_{1}=\Gamma^{-1}$. The quantity

$$
m_{2} b=u^{3 / 2}(1-3 u)^{1 / 2}\left(1+q \delta_{b}(u)\right),
$$

with

$$
\begin{aligned}
\delta_{b}(u) & =-\frac{m_{2}(1-4 u)}{4 u^{2}(1-3 u)} \partial_{r} h_{k k}^{(0)}+\frac{1}{u^{1 / 2}} \partial_{[r} h_{\phi] k}^{(0)} \\
& -\frac{1}{2}(1-2 u) h_{r r}^{(0)}-\frac{u}{2(1-3 u)(1-2 u)} h_{k k}^{(0)} \\
& -\frac{u^{3 / 2}}{m_{2}(1-2 u)}\left(h_{t \phi}^{(0)}+\frac{u^{1 / 2}(1-u)}{2 m_{2}} h_{\phi \phi}^{(0)}\right),
\end{aligned}
$$

is related to the spin precession frequency. In fact, the previous equation can be rewritten as

$$
\delta_{b}(u)=-\frac{m_{2}(1-4 u)}{4 u^{2}(1-3 u)} \partial_{r} h_{k k}^{(0)}-\delta(u),
$$

where $\delta(u)$ was defined in Eq. (3.9) of Ref. [46]. Passing to the variable $y$, the quantity $\delta_{b}(y)$ simply becomes $\delta_{b}(y)=-\delta(y)$. A direct comparison with Eq. (4.8) of Ref. [47] then leads to the identification $b=\Omega-\Omega_{1}$, where $\Omega$ is the orbital frequency and $\Omega_{1}$ the precession frequency. It is also related to the spin precession angle $\psi$ by $b=\Omega \psi$ (see Ref. [48]).

\section{GSF CORRECTIONS TO TIDAL INVARIANTS IN THE PRESENCE OF SPIN}

In any given spacetime the electric-tidal forces (relative to $U$ ) are governed by the "potentials"

$$
\begin{aligned}
\operatorname{Tr}\left[\mathcal{E}(U)^{2}\right] & =\mathcal{E}(U)_{\alpha \beta} \mathcal{E}(U)^{\alpha \beta}, \\
\operatorname{Tr}\left[\mathcal{E}(U)^{3}\right] & =\mathcal{E}(U)_{\alpha \beta} \mathcal{E}(U)^{\beta \mu} \mathcal{E}(U)_{\mu}{ }^{\alpha},
\end{aligned}
$$

where

$$
\mathcal{E}(U)_{\alpha \beta}=R_{\alpha \mu \beta \nu} U^{\mu} U^{\nu} .
$$

Similarly, the magnetic-tidal forces are governed by the potential

$$
\operatorname{Tr}\left[\mathcal{B}(U)^{2}\right]=\mathcal{B}(U)_{\alpha \beta} \mathcal{B}(U)^{\alpha \beta}
$$

where

$$
\mathcal{B}(U)_{\alpha \beta}=R^{*}{ }_{\alpha \mu \beta \nu} U^{\mu} U^{\nu} .
$$


Here $U=\Gamma k$ (with $\Gamma=U^{t}$ ) is the four velocity of the spinning particle and is aligned with a nongeodesic orbit (differently from previous works) because of the nonvanishing spin of the particle which implies the existence of spin-curvature coupling forces. Expanding $\Gamma$ and $\Omega$ in powers of $\hat{s}$ we find (at the first order in $\hat{s}$ )

$$
\begin{aligned}
& \Gamma=\bar{\Gamma}+q\left(\Gamma_{1}+\hat{s} \Gamma_{1 \hat{s}}\right), \\
& \Omega=\bar{\Omega}+q\left(\Omega_{1}+\hat{s} \Omega_{1 \hat{s}}\right),
\end{aligned}
$$

where

$$
\begin{aligned}
\bar{\Gamma} & =\frac{1}{\sqrt{1-3 u}}\left(1-\frac{3}{2} \hat{s} \frac{u^{5 / 2}}{1-3 u}\right), \\
m_{2} \bar{\Omega} & =u^{3 / 2}\left(1-\frac{3}{2} \hat{s} u^{3 / 2}\right) .
\end{aligned}
$$

The first-order self-force (1SF) corrections to the frequency $\Omega_{1}=u^{3 / 2} \tilde{\Omega}_{1}$ and $\Omega_{1 \hat{s}}=u^{3 / 2} \tilde{\Omega}_{1 \hat{s}}$ are given in Eqs. (2.13) (2.14) above, whereas the correction to the redshift factor $\Gamma=U^{t}=1 / z_{1}$ can be found in Ref. [41].

Let us consider the electric and magnetic part of the Riemann tensor with respect to $k$ instead of $U$ [36]. We compute both the quadratic tidal-electric and tidalmagnetic invariants as well as the cubic tidal-electric invariant defined by

$$
\begin{aligned}
\mathcal{J}_{e^{2}} & \equiv \operatorname{Tr}\left[\mathcal{E}^{2}(k)\right]=\mathcal{E}^{\mu}{ }_{\nu}(k) \mathcal{E}^{\nu}{ }_{\mu}(k) \\
\mathcal{J}_{b^{2}} & \equiv \operatorname{Tr}\left[\mathcal{B}^{2}(k)\right]=\mathcal{B}^{\mu}{ }_{\nu}(k) \mathcal{B}^{\nu}{ }_{\mu}(k) \\
\mathcal{J}_{e^{3}} & \equiv \operatorname{Tr}\left[\mathcal{E}^{3}(k)\right]=\mathcal{E}^{\mu}{ }_{\nu}(k) \mathcal{E}^{\nu}{ }_{\rho}(k) \mathcal{E}^{\rho}{ }_{\mu}(k),
\end{aligned}
$$

respectively, where

$$
\begin{aligned}
& \mathcal{E}_{\alpha \beta}(k) \equiv R_{\alpha \mu \beta \nu} k^{\mu} k^{\nu}=\Gamma^{-2} \mathcal{E}_{\alpha \beta}(U), \\
& \mathcal{B}_{\alpha \beta}(k) \equiv R_{\alpha \mu \beta \nu}^{*} k^{\mu} k^{\nu}=\Gamma^{-2} \mathcal{B}_{\alpha \beta}(U)
\end{aligned}
$$

The 1SF-accurate expansion of the $m_{2^{-}}$ adimensionalized version of both electric-like and magnetic-like tidal invariants, expressed in terms of $y$, reads

$$
\begin{aligned}
\tilde{\mathcal{J}}_{e^{2}}(y) & \equiv m_{2}^{4} \mathcal{J}_{e^{2}}=\tilde{\mathcal{J}}_{e^{2}}^{(0)}\left[1+q \delta_{e^{2}}(y)+O\left(q^{2}\right)\right] \\
\tilde{\mathcal{J}}_{b^{2}}(y) & \equiv m_{2}^{4} \mathcal{J}_{b^{2}}=\tilde{\mathcal{J}}_{b^{2}}^{(0)}\left[1+q \delta_{b^{2}}(y)+O\left(q^{2}\right)\right] \\
\tilde{\mathcal{J}}_{e^{3}}(y) & \equiv m_{2}^{6} \mathcal{J}_{e^{3}}=\tilde{\mathcal{J}}_{e^{3}}^{(0)}\left[1+q \delta_{e^{3}}(y)+O\left(q^{2}\right)\right]
\end{aligned}
$$

where

$$
\begin{aligned}
\tilde{\mathcal{J}}_{e^{2}}^{(0)}= & 6 y^{6}\left(1-3 y+3 y^{2}\right)+36 y^{15 / 2}(1-2 y)^{2} \hat{s} \\
\tilde{\mathcal{J}}_{b^{2}}^{(0)}= & 18 y^{7}(1-2 y)+36 y^{17 / 2}(2-5 y) \hat{s} \\
\tilde{\mathcal{J}}_{e^{3}}^{(0)}= & -3 y^{9}(1-3 y)(2-3 y) \\
& -27 y^{21 / 2}(1-2 y)(1-3 y)(2-y) \hat{s} .
\end{aligned}
$$

The GSF corrections $\delta_{e^{2}}, \delta_{b^{2}}$ and $\delta_{e^{3}}$ are understood to be regularized following the standard GSF procedure (which we do not need to repeat here) by subtracting their singular parts (here the " $B$-term"). ${ }^{1}$ In this way one is left with convergent series, e.g.,

$$
\delta^{\mathrm{reg}}=\sum_{l=0}^{\infty}\left(\delta_{l}^{0}-B(y ; l)\right)
$$

where

$$
\delta_{l}^{0} \equiv \frac{1}{2}\left(\delta_{l}^{+}+\delta_{l}^{-}\right)
$$

the "subtraction term" or "B-term" is of the form

$$
B(y ; l)=l(l+1) b_{0}(y)+b_{1}(y)
$$

with $b_{0}(y)=b_{0}^{\hat{s}^{0}}(y)+\hat{s} b_{0}^{\hat{s}^{1}}(y)$ and similarly for $b_{1}(y)$.

To make a long story short, we expand the metric perturbations in tensorial spherical harmonics in the ReggeWheeler gauge 49] following the original approach of Zerilli [50]. We solve then the resulting radial equations in PN-sense and use up to a fixed PN-order. To reach the latter order is necessary to combine $\mathrm{PN}$ type solutions with Mano-Suzuki-Takasugi (MST) type solutions [51, 52], following a standard approach first introduced in Ref. [53].

We will use here the MST solutions for the multipoles $l=2, \ldots, 7$, so that our result will be accurate up to the order $O\left(y^{19 / 2}\right)$ included, corresponding to the $9.5 \mathrm{PN}$ order.

\section{A. Tidal-electric: $\operatorname{Tr}\left[\mathcal{E}^{2}(k)\right]$}

The $O(q)$ perturbation to $\operatorname{Tr}\left[\mathcal{E}^{2}(k)\right]$ is given by the following combination of perturbed metric components $h_{\mu \nu}$ and their derivatives

$$
\delta_{e^{2}}(y)=\delta_{e^{2}, h}(y)+\delta_{e^{2}, \partial h}(y)+\delta_{e^{2}, \partial^{2} h}(y),
$$

where 
1 Actually the computation of the Detweiler-Whiting full singular field would require additional regularization parameters (see,

$$
\begin{aligned}
& \delta_{e^{2}, h}(y)=\left[-\frac{y}{3(-1+2 y)\left(1-3 y+3 y^{2}\right)}+\hat{s} \frac{y^{3 / 2}\left(15 y^{3}-105 y^{4}+90 y^{5}+30 y^{2}-17 y+3\right)}{3(-1+2 y)^{2}\left(1-3 y+3 y^{2}\right)^{2}}\right] h_{k k} \\
& +\left[\frac{(-1+2 y)\left(18 y^{2}-18 y+5\right)}{3\left(1-3 y+3 y^{2}\right)}+\hat{s} \frac{\left(594 y^{5}-1341 y^{4}+1293 y^{3}-657 y^{2}+172 y-18\right) y^{3 / 2}}{3\left(1-3 y+3 y^{2}\right)^{2}}\right] h_{r r} \\
& +\left[-\frac{y^{2}(3 y-1)(y-1)}{3(-1+2 y)\left(1-3 y+3 y^{2}\right) M^{2}}\right. \\
& \left.-\hat{s} \frac{\left(3033 y^{5}-1989 y^{6}+594 y^{7}-2643 y^{4}+1397 y^{3}-445 y^{2}+79 y-6\right) y^{5 / 2}}{3 M^{2}(-1+2 y)^{2}\left(1-3 y+3 y^{2}\right)^{2}}\right] h_{\phi \phi} \\
& +\left[-\frac{y^{2}}{3\left(1-3 y+3 y^{2}\right) M^{2}}+\hat{s} \frac{2\left(18 y^{3}-18 y^{2}+6 y-1\right) y^{7 / 2}}{3 M^{2}\left(1-3 y+3 y^{2}\right)^{2}}\right] h_{\theta \theta} \\
& +\left[\frac{2 y^{3 / 2}(3 y-1)}{3 M(-1+2 y)\left(1-3 y+3 y^{2}\right)}-\hat{s} \frac{4(3 y-2) y^{5}}{\left(1-3 y+3 y^{2}\right)^{2} M}\right] h_{t \phi} \\
& \delta_{e^{2}, \partial h}(y)=\left[\frac{M(3 y-1)(3 y-2)}{3 y^{2}\left(1-3 y+3 y^{2}\right)}+\hat{s} \frac{M\left(225 y^{2}-333 y^{3}+225 y^{4}+10-78 y\right)}{6 y^{1 / 2}\left(1-3 y+3 y^{2}\right)^{2}}\right] \partial_{r} h_{k k} \\
& +\left[\frac{(3 y-1) y}{3 M\left(1-3 y+3 y^{2}\right)}+\hat{s} \frac{y^{3 / 2}\left(243 y^{5}-783 y^{4}+888 y^{3}-471 y^{2}+121 y-12\right)}{6\left(1-3 y+3 y^{2}\right)^{2} M}\right] \partial_{r} h_{\phi \phi} \\
& +\left[\frac{(3 y-1)}{3 y^{1 / 2}\left(1-3 y+3 y^{2}\right)}-\hat{s} \frac{\left(3 y^{2}+3 y-1\right)\left(27 y^{3}-36 y^{2}+17 y-3\right)}{3\left(1-3 y+3 y^{2}\right)^{2}}\right] \partial_{r} h_{t \phi} \\
& +\left[-\frac{(3 y-1) y}{3 M\left(1-3 y+3 y^{2}\right)}-\hat{s} \frac{y^{3 / 2}\left(6 y^{2}-6 y+1\right)\left(18 y^{3}-33 y^{2}+17 y-3\right)}{3 M\left(1-3 y+3 y^{2}\right)^{2}}\right] \partial_{\phi} h_{r \phi} \\
& +\left[-\frac{(3 y-1)}{3 y^{1 / 2}\left(1-3 y+3 y^{2}\right)}-\hat{s} \frac{\left(27 y^{5}-171 y^{4}+228 y^{3}-126 y^{2}+32 y-3\right)}{3\left(1-3 y+3 y^{2}\right)^{2}}\right] \partial_{\phi} h_{t r} \\
& +\hat{s} \frac{(-1+2 y) y^{5 / 2}}{M\left(1-3 y+3 y^{2}\right)}\left(\partial_{\theta} h_{r \theta}-\partial_{r} h_{\theta \theta}\right) \\
& -\hat{s} \frac{(-1+2 y)^{2}\left(15 y^{2}-12 y+2\right)}{2 y^{1 / 2}\left(1-3 y+3 y^{2}\right)} \partial_{r} h_{r r} \\
& \delta_{e^{2}, \partial^{2} h}(y)=\left[\frac{(-1+2 y)(3 y-2) M^{2}}{6 y^{3}\left(1-3 y+3 y^{2}\right)}+\hat{s} \frac{M^{2}\left(-1+9 y-33 y^{2}+36 y^{3}\right)}{6 y^{1 / 2}\left(1-3 y+3 y^{2}\right)^{2}}\right] \partial_{r r} h_{k k} \\
& +\left[-\frac{1}{6\left(-3 y+1+3 y^{2}\right) y}+\hat{s} \frac{\left(18 y^{3}-9 y^{2}-3 y+1\right) y^{1 / 2}}{6\left(-3 y+1+3 y^{2}\right)^{2}}\right] \partial_{\theta \theta} h_{k k} \\
& +\left[\frac{(3 y-1)^{2}}{6\left(-3 y+1+3 y^{2}\right)(-1+2 y) y}+\hat{s} \frac{y^{1 / 2}\left(24 y^{3}-33 y^{2}+13 y-1\right)(3 y-1)^{2}}{6(-1+2 y)^{2}\left(-3 y+1+3 y^{2}\right)^{2}}\right] \partial_{\phi \phi} h_{k k} \\
& -\hat{s}\left[\frac{(3 y-1)(-1+2 y)^{2} M}{\left(-3 y+1+3 y^{2}\right) y}\right]\left(\partial_{r r} h_{\phi k}-\partial_{r \phi} h_{r k}\right) \text {. }
\end{aligned}
$$

The regularized value of the zeroth order in spin correction $\delta_{e^{2}}^{\hat{s}^{0}}(y)$ is known [36 39]. We recall below for completeness only the first few PN terms

$$
\begin{aligned}
\delta_{e^{2}}^{\hat{s}^{0}}(y) & =-2+5 y+\frac{61}{4} y^{2}+\left(\frac{593}{256} \pi^{2}-\frac{1669}{24}\right) y^{3} \\
& +\left(\frac{1118879}{4800}-\frac{5867}{1024} \pi^{2}-\frac{768}{5} \gamma-\frac{1536}{5} \ln (2)-\frac{384}{5} \ln (y)\right) y^{4}+O_{\ln }\left(y^{5}\right) .
\end{aligned}
$$

The correction linear in spin is instead new and given by 


$$
\begin{aligned}
& \delta_{e^{2}}^{\hat{s}^{1}}(y)=-2 y^{3 / 2}+15 y^{5 / 2}-\frac{107}{4} y^{7 / 2}+\left(-\frac{103}{24}+\frac{41}{16} \pi^{2}\right) y^{9 / 2} \\
& +\left(-\frac{292027}{576}+\frac{68867}{1536} \pi^{2}+\frac{1576}{15} \ln (y)+432 \ln (2)+\frac{3152}{15} \gamma\right) y^{11 / 2} \\
& +\left(-\frac{6751671}{3200}-\frac{42008}{35} \ln (2)+\frac{3764}{35} \ln (y)+\frac{7528}{35} \gamma+\frac{10935}{7} \ln (3)+\frac{172241}{8192} \pi^{2}\right) y^{13 / 2} \\
& +\frac{7704}{35} \pi y^{7} \\
& +\left(\frac{771338065013}{10160640}-\frac{6674991929}{884736} \pi^{2}-\frac{1037367}{70} \ln (3)+\frac{4392574}{567} \ln (2)-\frac{19876714}{2835} \gamma-\frac{9938357}{2835} \ln (y)\right. \\
& \left.+\frac{8432821}{131072} \pi^{4}\right) y^{15 / 2} \\
& +\frac{166681}{2205} \pi y^{8} \\
& +\left(\frac{703288938133497911}{1760330880000}+\frac{2606489917}{1212750} \ln (y)+\frac{2606489917}{606375} \gamma+\frac{491929443}{12320} \ln (3)+\frac{712890625}{28512} \ln (5)\right. \\
& -\frac{766912112971}{5457375} \ln (2)-\frac{11357408}{1575} \ln (2)^{2}-\frac{2811104}{1575} \gamma^{2}-\frac{702776}{157} 5 \ln (y)^{2}+\frac{52544}{15} \zeta(3) \\
& -\frac{844288199909}{402653184} \pi^{4}-\frac{74442851762681}{4954521600} \pi^{2}-\frac{3773248}{525} \gamma \ln (2) \\
& \left.-\frac{1886624}{525} \ln (2) \ln (y)-\frac{2811104}{1575} \gamma \ln (y)\right) y^{17 / 2} \\
& -\frac{4923636673}{727650} \pi y^{9} \\
& +\left(\frac{22417444716344159237593}{28193459374080000}+\frac{26271208759907}{29429400} \ln (2)+\frac{11228736392577}{35672000} \ln (3)\right. \\
& +\frac{159032483612257}{1589187600} \ln (y)+\frac{159479998840417}{794593800} \gamma-\frac{852930}{49} \ln (3)^{2}-\frac{646076171875}{1729728} \ln (5) \\
& +\frac{303921248}{11025} \ln (2)^{2}-\frac{69102496}{11025} \gamma^{2}-\frac{17275624}{11025} \ln (y)^{2} \\
& +\frac{237056}{7} \zeta(3)-\frac{5973531733489}{536870912} \pi^{4}-\frac{948482819940703}{277453209600} \pi^{2}+\frac{110477504}{11025} \gamma \ln (2) \\
& +\frac{55238752}{11025} \ln (2) \ln (y)-\frac{69102496}{11025} \gamma \ln (y)-\frac{1705860}{49} \gamma \ln (3)-\frac{1705860}{49} \ln (2) \ln (3) \\
& \left.-\frac{852930}{49} \ln (3) \ln (y)\right) y^{19 / 2}+O_{\ln }\left(y^{10}\right) \text {. }
\end{aligned}
$$

\section{B. Tidal-electric-cube: $\operatorname{Tr}\left[\mathcal{E}^{3}(k)\right]$}

The $O(q)$ perturbation to $\operatorname{Tr}\left[\mathcal{E}^{3}(k)\right]$ is given by $\delta_{e^{3}}(y)$ which has a formal expression in terms of the metric components similar to $\delta_{e^{2}}(y)$. The regularized value of the zeroth order in spin correction $\delta_{e^{3}}^{\hat{s}^{0}}(y)$ is known [36 [39]. We recall below for completeness only the first few PN terms

$$
\begin{aligned}
\delta_{e^{3}}^{\hat{s}^{3}}(y)= & -3+\frac{15}{2} y+\frac{147}{8} y^{2}+\left(-\frac{1561}{16}+\frac{1779}{512} \pi^{2}\right) y^{3} \\
& +\left(\frac{1336679}{3200}-\frac{2271}{256} \pi^{2}-\frac{1152}{5} \gamma-\frac{2304}{5} \ln (2)-\frac{576}{5} \ln (y)\right) y^{4}+O_{\ln }\left(y^{5}\right) .
\end{aligned}
$$

The correction linear in spin is instead new and given by 


$$
\begin{aligned}
& \delta_{e^{3}}^{\hat{s}^{1}}(y)=-3 y^{3 / 2}+\frac{45}{2} y^{5 / 2}-\frac{105}{8} y^{7 / 2}+\left(-\frac{535}{16}+\frac{123}{32} \pi^{2}\right) y^{9 / 2} \\
& +\left(-\frac{371947}{384}+\frac{138301}{2048} \pi^{2}+\frac{1576}{5} \gamma+648 \ln (2)+\frac{788}{5} \ln (y)\right) y^{11 / 2} \\
& +\left(-\frac{34633509}{6400}+\frac{702285}{4096} \pi^{2}+\frac{34224}{35} \gamma-\frac{17232}{35} \ln (2)+\frac{17112}{35} \ln (y)+\frac{32805}{14} \ln (3)\right) y^{13 / 2} \\
& +\frac{11556}{35} \pi y^{7} \\
& +\left(\frac{2406372381697}{33868800}+\frac{2274533}{189} \ln (2)-\frac{1310013}{70} \ln (3)-\frac{8095607}{1890} \ln (y)-\frac{8095607}{945} \gamma-\frac{9113917597}{1179648} \pi^{2}\right. \\
& \left.+\frac{25298463}{262144} \pi^{4}\right) y^{15 / 2} \\
& +\frac{1146373}{1470} \pi y^{8} \\
& +\left(\frac{594053200308222311}{1173553920000}-\frac{1886624}{175} \gamma \ln (2)-\frac{943312}{175} \ln (2) \ln (y)-\frac{1405552}{525} \gamma \ln (y)-\frac{824612241077}{268435456} \pi^{4}\right. \\
& -\frac{22341281351653}{1651507200} \pi^{2}-\frac{1405552}{525} \gamma^{2}-\frac{5678704}{525} \ln (2)^{2}-\frac{351388}{525} \ln (y)^{2}+\frac{26272}{5} \zeta(3)-\frac{369489776273}{1819125} \ln (2) \\
& \left.-\frac{1443347929}{404250} \ln (y)-\frac{1443347929}{202125} \gamma+\frac{712890625}{19008} \ln (5)+\frac{976942377}{24640} \ln (3)\right) y^{17 / 2} \\
& -\frac{2053395569}{242550} \pi y^{9} \\
& +\left(\frac{49851744177689427024889}{18795639582720000}-\frac{23028752}{3675} \gamma \ln (2)-\frac{11514376}{3675} \ln (2) \ln (y)-\frac{54127112}{3675} \gamma \ln (y)\right. \\
& -\frac{2558790}{49} \gamma \ln (3)-\frac{2558790}{49} \ln (2) \ln (3)-\frac{1279395}{49} \ln (3) \ln (y)-\frac{9399098116257}{335544320} \pi^{4}-\frac{11234318687460683}{369937612800} \pi^{2} \\
& -\frac{54127112}{3675} \gamma^{2}+\frac{73711096}{3675} \ln (2)^{2}-\frac{13531778}{3675} \ln (y)^{2}+\frac{2143824}{35} \zeta(3)+\frac{1000473336848339}{882882000} \ln (2) \\
& +\frac{759486010098089}{5297292000} \ln (y)+\frac{761723586238889}{2648646000} \gamma-\frac{585646484375}{1153152} \ln (5)-\frac{1279395}{49} \ln (3)^{2} \\
& \left.+\frac{370377504565641}{784784000} \ln (3)\right) y^{19 / 2}+O_{\ln }\left(y^{10}\right) \text {. }
\end{aligned}
$$

\section{Tidal-magnetic: $\operatorname{Tr}\left[\mathcal{B}^{2}(k)\right]$}

The $O(q)$ perturbation to $\operatorname{Tr}\left[\mathcal{B}^{2}(k)\right]$ is given by $\delta_{b^{2}}(y)$ which has a formal expression in terms of the metric components similar to $\delta_{e^{2}}(y)$. The regularized value of the zeroth order in spin correction $\delta_{b^{2}}^{\hat{s}^{0}}(y)$ is known [36 [39]. We recall below for completeness only the first few PN terms

$$
\begin{aligned}
\delta_{b^{2}}^{\hat{s}^{0}}(y)= & -\frac{4}{3}+\frac{14}{3} y-\frac{11}{6} y^{2}+\left(-\frac{1723}{36}+\frac{41}{24} \pi^{2}\right) y^{3} \\
& +\left(-\frac{357079}{4320}+\frac{73559}{4608} \pi^{2}-\frac{3616}{45} \gamma-160 \ln (2)-\frac{1808}{45} \ln (y)\right) y^{4}+O_{\ln }\left(y^{5}\right) .
\end{aligned}
$$

The correction linear in spin is instead new and given by 


$$
\begin{aligned}
& \delta_{b^{2}}^{\hat{s}^{1}}(y)=-\frac{4}{3} y^{3 / 2}+\frac{37}{3} y^{5 / 2}+\left(-19+\frac{63}{1024} \pi^{2}\right) y^{7 / 2} \\
& +\left(-\frac{62711}{1800}+\frac{1081}{768} \pi^{2}-\frac{8}{15} \ln (y)-\frac{16}{15} \ln (2)-\frac{16}{15} \gamma\right) y^{9 / 2} \\
& +\left(-\frac{222888751}{151200}+\frac{87754979}{589824} \pi^{2}+\frac{2392}{45} \gamma+\frac{6728}{63} \ln (2)+\frac{1196}{45} \ln (y)\right) y^{11 / 2} \\
& -\frac{856}{1575} \pi y^{6} \\
& +\left(\frac{1727123297}{604800}-\frac{267590669}{524288} \pi^{2}+\frac{39206}{105} \gamma+\frac{40058}{135} \ln (2)+\frac{19603}{105} \ln (y)\right. \\
& \left.+\frac{2916}{7} \ln (3)+\frac{26973279}{2097152} \pi^{4}\right) y^{13 / 2} \\
& +\frac{8636}{147} \pi y^{7} \\
& +\left(\frac{1802670191688469}{18336780000}-\frac{2224682621317007}{237817036800} \pi^{2}-\frac{223317}{77} \ln (3)+\frac{4484235971}{16372125} \ln (2)-\frac{3246009239}{1488375} \gamma\right. \\
& -\frac{3246009239}{2976750} \ln (y)-\frac{86776713349}{1610612736} \pi^{4}+\frac{3424}{1575} \ln (2)^{2}+\frac{3424}{1575} \gamma \ln (y) \\
& \left.+\frac{6848}{1575} \gamma \ln (2)+\frac{3424}{1575} \ln (2) \ln (y)-\frac{64}{15} \zeta(3)+\frac{856}{1575} \ln (y)^{2}+\frac{3424}{1575} \gamma^{2}\right) y^{15 / 2} \\
& +\frac{7531774}{24255} \pi y^{8} \\
& +\left(\frac{14620969720956075313}{14562737280000}+\frac{841954257}{254800} \ln (3)-\frac{2607400020943}{1833678000} \ln (y)-\frac{4849325459983}{154791000} \ln (2)\right. \\
& -\frac{2669989563343}{916839000} \gamma+\frac{1005859375}{185328} \ln (5)+\frac{269728}{315} \zeta(3)-\frac{15577328}{33075} \gamma^{2}-\frac{62406896}{33075} \ln (2)^{2} \\
& -\frac{3894332}{33075} \ln (y)^{2}-\frac{2970208}{1575} \gamma \ln (2)-\frac{1485104}{1575} \ln (2) \ln (y)-\frac{15577328}{33075} \gamma \ln (y) \\
& \left.-\frac{2756098577896711}{369937612800} \pi^{2}-\frac{7228107040550893}{773094113280} \pi^{4}\right) y^{17 / 2} \\
& +\left(-\frac{21795132889051}{9833098275} \pi+\frac{366368}{165375} \gamma \pi+\frac{366368}{165375} \pi \ln (2)-\frac{3424}{4725} \pi^{3}+\frac{183184}{165375} \pi \ln (y)\right) y^{9} \\
& +\left(-\frac{415840188382430911927073}{465192079672320000}+\frac{148196991257948671}{786647862000} \ln (2)+\frac{11975992669593}{134884750} \ln (3)\right. \\
& +\frac{3145694346466161199}{131941395333120} \pi^{4}-\frac{62764648557015325903}{1171962357350400} \pi^{2}-\frac{1101472088331}{1073741824} \pi^{6} \\
& -\frac{4382214476}{1091475} \gamma^{2}-\frac{2166015625}{30888} \ln (5)-\frac{12928086}{2695} \ln (3)^{2} \\
& +\frac{55360630827270719}{1573295724000} \ln (y)+\frac{875944}{63} \zeta(3)-\frac{1597686092}{1091475} \ln (2)^{2} \\
& +\frac{55642565421011519}{786647862000} \gamma-\frac{1095553619}{1091475} \ln (y)^{2}-\frac{25856172}{2695} \ln (2) \ln (3)-\frac{25856172}{2695} \gamma \ln (3) \\
& \left.-\frac{2302692232}{363825} \gamma \ln (2)-\frac{1151346116}{363825} \ln (2) \ln (y)-\frac{12928086}{2695} \ln (3) \ln (y)-\frac{4382214476}{1091475} \gamma \ln (y)\right) y^{19 / 2} \\
& +O_{\ln }\left(y^{10}\right) \text {. }
\end{aligned}
$$

\section{EIGENVALUES}

quadrupolar tensors $m_{2}^{2} \mathcal{E}^{\mu}{ }_{\nu}(U), m_{2}^{2} \mathcal{B}^{\mu}{ }_{\nu}(U)$. These eigen-
We compute below the $1 \mathrm{SF}$ contribution to the eigenvalues of the tidal-electric, and tidal-magnetic, 
values are such that

$$
\begin{aligned}
& m_{2}^{2} \mathcal{E}(U)=\operatorname{diag}\left[\lambda_{1}^{(\mathrm{E})}, \lambda_{2}^{(\mathrm{E})},-\left(\lambda_{1}^{(\mathrm{E})}+\lambda_{2}^{(\mathrm{E})}\right)\right] \\
& m_{2}^{2} \mathcal{B}(U)=\operatorname{diag}\left[\lambda^{(\mathrm{B})},-\lambda^{(\mathrm{B})}, 0\right],
\end{aligned}
$$

where we used their tracelessness, and the existence of a zero eigenvalue of $\mathcal{B}(U)$ [37]. Let us introduce a notation for the eigenvalues of the corresponding rescaled tidal tensors

$$
\begin{aligned}
& m_{2}^{2} \mathcal{E}(k)=\operatorname{diag}\left[\sigma_{1}^{(\mathrm{E})}, \sigma_{2}^{(\mathrm{E})},-\left(\sigma_{1}^{(\mathrm{E})}+\sigma_{2}^{(\mathrm{E})}\right)\right] \\
& m_{2}^{2} \mathcal{B}(k)=\operatorname{diag}\left[\sigma^{(\mathrm{B})},-\sigma^{(\mathrm{B})}, 0\right],
\end{aligned}
$$

evaluated with respect to $k$ instead of $U$. The two set of eigenvalues are related by

$$
\lambda_{a}^{(\mathrm{E})}=\Gamma^{2} \sigma_{a}^{(\mathrm{E})}, \quad \lambda^{(\mathrm{B})}=\Gamma^{2} \sigma^{(\mathrm{B})},
$$

where the 1SF expansion of the redshift factor

$$
\Gamma=\frac{1}{\sqrt{1-3 y}}-q \frac{z_{1}^{1 \mathrm{SF}}(y)}{1-3 y},
$$

has been derived to first order in spin in our previous work [41]. One finds

$$
\begin{aligned}
& \lambda_{1}^{(\mathrm{E})}=\lambda_{1}^{(\mathrm{E}) 0 \mathrm{SF}}+q \lambda_{1}^{(\mathrm{E}) 1 \mathrm{SF}}, \\
& \lambda_{2}^{(\mathrm{E})}=\lambda_{2}^{(\mathrm{E}) 0 \mathrm{SF}}+q \lambda_{2}^{(\mathrm{E}) 1 \mathrm{SF}}, \\
& \lambda^{(\mathrm{B})}=\lambda^{(\mathrm{B}) 0 \mathrm{SF}}+q \lambda^{(\mathrm{B}) 1 \mathrm{SF}},
\end{aligned}
$$

where the unperturbed (0SF) values of these eigenvalues are given $b^{2}$

$$
\begin{aligned}
\lambda_{1}^{(\mathrm{E}) 0 \mathrm{SF}} & =-y^{3} \frac{2-3 y}{1-3 y}-3 \hat{s} y^{9 / 2} \frac{2-5 y}{1-3 y}, \\
\lambda_{2}^{(\mathrm{E}) 0 \mathrm{SF}} & =\frac{y^{3}}{1-3 y}+3 \hat{s} y^{9 / 2} \frac{1-2 y}{1-3 y}, \\
-\lambda^{(\mathrm{B}) 0 \mathrm{SF}} & =-3 y^{7 / 2} \frac{\sqrt{1-2 y}}{1-3 y}-\hat{s} \frac{3 y^{5}(2-5 y)}{\sqrt{1-2 y}(1-3 y)},
\end{aligned}
$$

and the 1SF corrections are

$$
\begin{aligned}
& \lambda_{1}^{(\mathrm{E}) 1 \mathrm{SF}}=\lambda_{1 \hat{s}^{0}}^{(\mathrm{E}) \mathrm{SF}}+\hat{s} \lambda_{1 \hat{s}^{1}}^{(\mathrm{E}) 1 \mathrm{SF}}, \\
& \lambda_{2}^{(\mathrm{E}) 1 \mathrm{SF}}=\lambda_{2 \hat{s}^{0}}^{(\mathrm{E}) 1 \mathrm{SF}}+\hat{s} \lambda_{2 \hat{s}^{1}}^{(\mathrm{E}) 1 \mathrm{SF}} \text {, } \\
& \lambda^{(\mathrm{B}) 1 \mathrm{SF}}=\lambda_{\hat{s}^{0}}^{(B) 1 S F}+\hat{s} \lambda_{\hat{s}^{1}}^{(\mathrm{B}) 1 \mathrm{SF}} \text {. }
\end{aligned}
$$

By introducing the notation [36]

$$
\begin{aligned}
& \alpha_{1 \mathrm{SF}}=\frac{1}{2} \tilde{\mathcal{J}}_{e^{2}}^{(0)} \delta_{e^{2}}(y), \\
& \beta_{1 \mathrm{SF}}=\frac{1}{3} \tilde{\mathcal{J}}_{e^{3}}^{(0)} \delta_{e^{3}}(y),
\end{aligned}
$$

the $1 \mathrm{SF}$ perturbation of the exact equations

$$
\begin{aligned}
& \frac{1}{2} m_{2}^{4} \operatorname{Tr}\left[\mathcal{E}^{2}(k)\right]=\sigma_{1}^{(\mathrm{E})_{2}}+\sigma_{2}^{(\mathrm{E}) 2}+\sigma_{1}^{(\mathrm{E})} \sigma_{2}^{(\mathrm{E})}, \\
& \frac{1}{3} m_{2}^{6} \operatorname{Tr}\left[\mathcal{E}^{3}(k)\right]=-\sigma_{1}^{(\mathrm{E})} \sigma_{2}^{(\mathrm{E})}\left(\sigma_{1}^{(\mathrm{E})}+\sigma_{2}^{(\mathrm{E})}\right)
\end{aligned}
$$

yields a linear system of two equations for the two unknowns $\sigma_{1}^{(\mathrm{E}) 1 \mathrm{SF}}, \sigma_{2}^{(\mathrm{E}) 1 \mathrm{SF}}$ with $\alpha_{1 \mathrm{SF}}$ and $\beta_{1 \mathrm{SF}}$ as right hand sides. The (unique) solution of this system reads

$$
\begin{aligned}
\sigma_{1}^{(\mathrm{E}) 1 \mathrm{SF}} & =\frac{\alpha_{1 \mathrm{SF}} \sigma_{1}^{(\mathrm{E}) 0 \mathrm{SF}}+\beta_{1 \mathrm{SF}}}{\left(\sigma_{1}^{(\mathrm{E}) 0 \mathrm{SF}}-\sigma_{2}^{(\mathrm{E}) 0 \mathrm{SF}}\right)\left(2 \sigma_{1}^{(\mathrm{E}) 0 \mathrm{SF}}+\sigma_{2}^{(\mathrm{E}) 0 \mathrm{SF}}\right)}, \\
\sigma_{2}^{(\mathrm{E}) 1 \mathrm{SF}} & =\frac{\alpha_{1 \mathrm{SF}} \sigma_{2}^{(\mathrm{E}) 0 \mathrm{SF}}+\beta_{1 \mathrm{SF}}}{\left(\sigma_{2}^{(\mathrm{E}) 0 \mathrm{SF}}-\sigma_{1}^{(\mathrm{E}) 0 \mathrm{SF}}\right)\left(2 \sigma_{2}^{(\mathrm{E}) 0 \mathrm{SF}}+\sigma_{1}^{(\mathrm{E}) 0 \mathrm{SF}}\right)} .
\end{aligned}
$$

As already discussed in Ref. [36], we recall that the denominators $\left(2 \sigma_{1}^{(\mathrm{E}) 0 \mathrm{SF}}+\sigma_{2}^{(\mathrm{E}) 0 \mathrm{SF}}\right)$ and $\left(2 \sigma_{2}^{(\mathrm{E}) 0 \mathrm{SF}}+\sigma_{1}^{(\mathrm{E}) 0 \mathrm{SF}}\right)$ have different $\mathrm{PN}$ orders. Indeed, in the Newtonian limit $(y \rightarrow 0) \sigma_{1}^{(\mathrm{E}) 0 \mathrm{SF}} \simeq-2 y^{3}$ and $\sigma_{2}^{(\mathrm{E}) 0 \mathrm{SF}} \simeq+y^{3}$, so that $\left(2 \sigma_{1}^{(\mathrm{E}) 0 \mathrm{SF}}+\sigma_{2}^{(\mathrm{E}) 0 \mathrm{SF}}\right) \simeq-3 y^{3}$, while $\left(2 \sigma_{2}^{(\mathrm{E}) 0 \mathrm{SF}}+\right.$ $\left.\sigma_{1}^{(\mathrm{E}) \mathrm{OSF}}\right)=O\left(y^{4}\right)$ is of $1 \mathrm{PN}$ fractional magnitude. As a consequence, one PN level in the analytic accuracy of $\sigma_{2}^{(\mathrm{E}) 0 \mathrm{SF}}$ is lost.

The 1SF correction to the tidal-magnetic eigenvalue $\lambda^{(\mathrm{B})}$ is simply evaluated as

$$
\sigma^{(\mathrm{B}) 1 \mathrm{SF}}=\frac{\tilde{\mathcal{J}}_{b^{2}}^{(0)} \delta_{b^{2}}(y)}{4 \sigma^{(\mathrm{B}) 0 S F}} .
$$

The regularized value of the zeroth order in spin corrections of the $U$-normalized eigenvalues $\lambda_{1 \hat{s}^{0}}^{(\mathrm{E})} \mathrm{SF}, \lambda_{2 \hat{s}^{0}}^{(\mathrm{E}) 1 \mathrm{SF}}$ and $\lambda_{\hat{s}^{0}}^{(\mathrm{B}) 1 \mathrm{SF}}$ are known [36 39$]$. We recall below for completeness only the first few PN terms 
2 These eigenvalues have been already computed in Ref. [19] (see Eq. (4.44) there), but appear misprinted.

$$
\begin{aligned}
\lambda_{1 \hat{s}^{0}}^{(\mathrm{E}) \mathrm{SF}}= & 2 y^{3}+2 y^{4}-\frac{19}{4} y^{5}+\left(\frac{227}{3}-\frac{593}{256} \pi^{2}\right) y^{6} \\
& +\left(-\frac{71779}{4800}-\frac{719}{256} \pi^{2}+\frac{1536}{5} \ln (2)+\frac{384}{5} \ln (y)+\frac{768}{5} \gamma\right) y^{7}+O_{\ln }\left(y^{8}\right) \\
\lambda_{2 \hat{s}^{0}}^{(\mathrm{E}) \mathrm{SF}}= & -y^{3}-\frac{3}{2} y^{4}-\frac{23}{8} y^{5}+\left(-\frac{2593}{48}+\frac{1249}{1024} \pi^{2}\right) y^{6} \\
& +\left(-\frac{362051}{3200}-\frac{128}{5} \ln (y)+\frac{1737}{1024} \pi^{2}-\frac{256}{5} \gamma-\frac{512}{5} \ln (2)\right) y^{7}+O_{\ln }\left(y^{8}\right) \\
-\lambda_{\hat{s}^{0}}^{(\mathrm{B}) 1 \mathrm{SF}}= & 2 y^{7 / 2}+3 y^{9 / 2}+\frac{59}{4} y^{11 / 2}+\left(\frac{2761}{24}-\frac{41}{16} \pi^{2}\right) y^{13 / 2} \\
& +\left(\frac{1618039}{2880}-\frac{112919}{3072} \pi^{2}+\frac{1808}{15} \gamma+240 \ln (2)+\frac{904}{15} \ln (y)\right) y^{15 / 2}+O_{\ln }\left(y^{17 / 2}\right) .
\end{aligned}
$$

The corrections linear in spin are given by

$$
\begin{aligned}
& \lambda_{1 \hat{s}^{1}}^{(\mathrm{E}) 1 \mathrm{SF}}=8 y^{9 / 2}-12 y^{11 / 2}-18 y^{13 / 2}+\left(\frac{659}{3}-\frac{2435}{256} \pi^{2}\right) y^{15 / 2} \\
& +\left(\frac{902941}{3600}-\frac{154267}{3072} \pi^{2}+\frac{752}{3} \gamma+\frac{2448}{5} \ln (2)+\frac{376}{3} \ln (y)\right) y^{17 / 2} \\
& +\left(\frac{55233761}{16800}-\frac{1272409}{8192} \pi^{2}-\frac{59176}{35} \gamma-\frac{29588}{35} \ln (y)-\frac{319352}{105} \ln (2)-\frac{2187}{7} \ln (3)\right) y^{19 / 2} \\
& +\frac{43656}{175} \pi y^{10} \\
& +\left(-\frac{63262994029}{793800}+\frac{71412166381}{7077888} \pi^{2}-\frac{443678}{405} \gamma-\frac{19495058}{2835} \ln (2)-\frac{221839}{405} \ln (y)+\frac{89667}{70} \ln (3)\right. \\
& \left.-\frac{60882449}{1048576} \pi^{4}\right) y^{21 / 2} \\
& -\frac{18887593}{11025} \pi y^{11} \\
& +\left(\frac{77805223927}{1819125} \gamma-\frac{3105568}{1575} \gamma^{2}+\frac{672258572747}{402653184} \pi^{4}+\frac{710346154789}{5457375} \ln (2)+\frac{16466409}{6160} \ln (3)\right. \\
& -\frac{2461856}{315} \ln (2)^{2}-\frac{224609375}{14256} \ln (5)+\frac{58048}{15} \zeta(3)+\frac{897434891715947}{19818086400} \pi^{2}-\frac{4115648}{525} \gamma \ln (2) \\
& \left.-\frac{9073265747546491}{13752585000}+\frac{77805223927}{3638250} \ln (y)-\frac{3105568}{1575} \gamma \ln (y)-\frac{2057824}{525} \ln (2) \ln (y)-\frac{776392}{1575} \ln (y)^{2}\right) y^{23 / 2} \\
& -\frac{1560074701}{2182950} \pi y^{12} \\
& +\left(-\frac{35676003719939}{361179000} \gamma+\frac{163272544}{11025} \gamma^{2}+\frac{676470994112801}{42949672960} \pi^{4}-\frac{1669871109946433}{3972969000} \ln (2)\right. \\
& -\frac{21959878162869}{196196000} \ln (3)+\frac{536078176}{11025} \ln (2)^{2}+\frac{170586}{49} \ln (3)^{2}+\frac{504291015625}{2594592} \ln (5)-\frac{230784}{7} \zeta(3) \\
& +\frac{1402717571812867}{10276044800} \pi^{2}+\frac{575082176}{11025} \gamma \ln (2)+\frac{341172}{49} \gamma \ln (3)+\frac{341172}{49} \ln (2) \ln (3) \\
& -\frac{35306156423939}{722358000} \ln (y)-\frac{4153593072625400734241}{1409672968704000}+\frac{163272544}{11025} \gamma \ln (y)+\frac{170586}{49} \ln (3) \ln (y) \\
& \left.+\frac{287541088}{11025} \ln (2) \ln (y)+\frac{40818136}{11025} \ln (y)^{2}\right) y^{25 / 2}+O_{\ln }\left(y^{13}\right),
\end{aligned}
$$




$$
\begin{aligned}
\lambda_{2 \hat{s}^{1}}^{(\mathrm{E}) 1 \mathrm{SF}}= & -4 y^{9 / 2}+2 y^{11 / 2}+\frac{9}{2} y^{13 / 2}+\left(-\frac{1627}{12}+\frac{2561}{512} \pi^{2}\right) y^{15 / 2} \\
& +\left(\frac{610013}{7200}-\frac{243815}{24576} \pi^{2}-\frac{2144}{15} \gamma-\frac{1072}{15} \ln (y)-\frac{4192}{15} \ln (2)\right) y^{17 / 2} \\
& +\left(\frac{85419731}{6720}-\frac{2819407}{2048} \pi^{2}+\frac{20796}{35} \gamma+\frac{10398}{35} \ln (y)+\frac{19244}{15} \ln (2)-\frac{729}{7} \ln (3)\right) y^{19 / 2} \\
& -\frac{224272}{1575} \pi y^{10} \\
& +\left(\frac{2892840540493}{25401600}-\frac{374039842301}{28311552} \pi^{2}+\frac{515194}{405} \gamma+\frac{4197526}{2835} \ln (2)+\frac{257597}{405} \ln (y)\right. \\
& \left.+\frac{43011}{35} \ln (3)+\frac{735266513}{8388608} \pi^{4}\right) y^{21 / 2} \\
& +\frac{2301737}{3675} \pi y^{11} \\
& +\left(-\frac{24978143909}{1559250} \gamma-\frac{24978143909}{3118500} \ln (y)+\frac{253376}{225} \gamma^{2}+\frac{879445089053}{1006632960} \pi^{4}\right. \\
& -\frac{5956778549}{198450} \ln (2)-\frac{5490099}{1540} \ln (3)+\frac{7032896}{1575} \ln (2)^{2}+\frac{9765625}{7128} \ln (5)-\frac{33152}{15} \zeta(3) \\
& -\frac{98262348401861}{1887436800} \pi^{2}+\frac{1410688}{315} \gamma \ln (2)+\frac{63344}{225} \ln (y)^{2}+\frac{54665096945177993}{125737920000} \\
& \left.+\frac{705344}{315} \ln (2) \ln (y)+\frac{253376}{225} \gamma \ln (y)\right) y^{23 / 2}+O_{\ln }\left(y^{12}\right)
\end{aligned}
$$




$$
\begin{aligned}
& -\lambda_{\hat{s}^{1}}^{(\mathrm{B}) \mathrm{SF}}=6 y^{5}-\frac{21}{2} y^{6}+\left(31-\frac{189}{2048} \pi^{2}\right) y^{7}+\left(\frac{97537}{400}-\frac{7599}{1024} \pi^{2}+\frac{4}{5} \ln (y)+\frac{8}{5} \ln (2)+\frac{8}{5} \gamma\right) y^{8} \\
& +\left(\frac{321104729}{100800}-\frac{117514627}{393216} \pi^{2}+\frac{1234}{15} \ln (y)+\frac{2468}{15} \gamma+\frac{33916}{105} \ln (2)\right) y^{9} \\
& +\frac{428}{525} \pi y^{19 / 2} \\
& +\left(\frac{1154329703}{403200}+\frac{313611845}{3145728} \pi^{2}-\frac{91853}{105} \gamma-\frac{511207}{315} \ln (2)-\frac{91853}{210} \ln (y)-\frac{729}{7} \ln (3)-\frac{80919837}{4194304} \pi^{4}\right) y^{10} \\
& +\frac{580942}{3675} \pi y^{21 / 2} \\
& +\left(-\frac{342516124855213}{1811040000}+\frac{1113494091353669}{52848230400} \pi^{2}-\frac{550915387}{330750} \gamma-\frac{550915387}{661500} \ln (y)-\frac{15669434057}{3638250} \ln (2)\right. \\
& -\frac{264141}{770} \ln (3)+\frac{32}{5} \zeta(3)-\frac{63982008699}{1073741824} \pi^{4}-\frac{3424}{525} \gamma \ln (2)-\frac{1712}{525} \gamma^{2}-\frac{1712}{525} \ln (2)^{2} \\
& \left.-\frac{428}{525} \ln (y)^{2}-\frac{1712}{525} \gamma \ln (y)-\frac{1712}{525} \ln (2) \ln (y)\right) y^{11} \\
& -\frac{105172666}{121275} \pi y^{23 / 2} \\
& +\left(\frac{14401009433479}{611226000} \gamma-\frac{1534056}{1225} \gamma^{2}+\frac{8191460400868013}{515396075520} \pi^{4}+\frac{9128850893003}{162162000} \ln (2)\right. \\
& +\frac{25951426299}{5605600} \ln (3)-\frac{6090856}{1225} \ln (2)^{2}-\frac{2001953125}{370656} \ln (5)+\frac{268784}{105} \zeta(3) \\
& +\frac{189103487160367811}{2219625676800} \pi^{2}-\frac{2616848}{525} \gamma \ln (2)-\frac{1534056}{1225} \gamma \ln (y)-\frac{768140015394994675117}{320380220160000} \\
& \left.-\frac{1308424}{525} \ln (2) \ln (y)+\frac{14338419891079}{1222452000} \ln (y)-\frac{383514}{1225} \ln (y)^{2}\right) y^{12} \\
& +\left(-\frac{362196722747}{262215954} \pi+\frac{1712}{1575} \pi^{3}-\frac{183184}{55125} \gamma \pi-\frac{91592}{55125} \pi \ln (y)-\frac{183184}{55125} \pi \ln (2)\right) y^{25 / 2} \\
& +\left(-\frac{2738651457992501}{74918844000} \gamma+\frac{3304416264993}{2147483648} \pi^{6}+\frac{382334818}{51975} \gamma^{2}+\frac{5177433837486038387}{263882790666240} \pi^{4}\right. \\
& -\frac{8316327878531317}{74918844000} \ln (2)-\frac{10245646667673}{269769500} \ln (3)+\frac{260978042}{10395} \ln (2)^{2}+\frac{3755079}{2695} \ln (3)^{2} \\
& +\frac{23521484375}{432432} \ln (5)-\frac{79044}{5} \zeta(3)+\frac{5703631259017453919}{15945066086400} \pi^{2}+\frac{459902188}{17325} \gamma \ln (2) \\
& +\frac{7510158}{2695} \gamma \ln (3)+\frac{7510158}{2695} \ln (2) \ln (3)+\frac{382334818}{51975} \gamma \ln (y)+\frac{3755079}{2695} \ln (3) \ln (y) \\
& +\frac{229951094}{17325} \ln (2) \ln (y)-\frac{2686867553019701}{149837688000} \ln (y)-\frac{14630621620415023880359}{2109714647040000} \\
& \left.+\frac{191167409}{103950} \ln (y)^{2}\right) y^{13}+O\left(y^{27 / 2}\right) .
\end{aligned}
$$

We show in Fig. 1 the behavior of the ratios between the 1SF first-order in spin contributions and the zeroth order ones $R_{1 e}=\lambda_{1 \hat{s}^{1}}^{(\mathrm{E}) 1 \mathrm{SF}} / \lambda_{1 \hat{s}^{0}}^{(\mathrm{E}) 1 \mathrm{SF}}, R_{2 e}=$ $\lambda_{2 \hat{s}^{1}}^{(\mathrm{E}) 1 \mathrm{SF}} / \lambda_{2}^{(\mathrm{E}) 1 \mathrm{SF}}$ and $R_{b}=\lambda_{\hat{s}^{1}}^{(\mathrm{B}) 1 \mathrm{SF}} / \lambda_{\hat{s}^{0}}^{(\mathrm{B}) 1 \mathrm{SF}}$ as functions of $y$. In the weak field region, well described by the PN approximation, their behavior is characterized by a general increasing. The maximum amount of these ratios is about $10 \%$ in the range $y \in[0,0.1]$, a value which is al- most doubled increasing the $y$-interval in $[0,0.2]$. Therefore, the contribution due to spin is in general smaller that $0.1 \hat{s}$ times the zeroth order term. Approaching the strong field region $(y \gtrsim 0.15)$ we see the onset of typical PN oscillations, whose physical meaning can be inferred only by significantly raising the accuracy theoretically (to very high-PN orders as in Ref. [38] for the spinless case) or by performing fully numerical analyses. Unfortunately, the lack in the literature of numerical studies of 


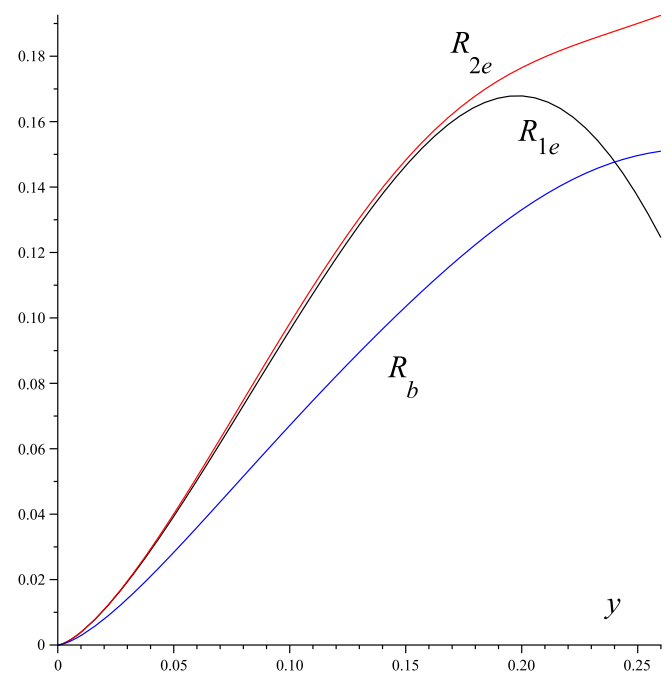

FIG. 1: The ratios $R_{1 e}, R_{2} e$ and $R_{b}$ between the 1SF firstorder in spin corrections to the electric and magnetic eigenvalues and the zeroth order ones are plotted as functions of $y$.

these quantities prevents us to have more insight in the strong field region.

\section{CONCLUDING REMARKS}

In this work we have analytically computed the $1 \mathrm{SF}$ corrections to the (quadratic and cubic) electric-type and (quadratic) magnetic-type tidal invariants for a binary system consisting of a nonrotating body (with larger mass $m_{2}$ ) and an extended body endowed with spin (with smaller mass $m_{1}$ ) to the first-order in spin and in the extreme-mass-ratio limit $m_{1} \ll m_{2}$, generalizing previous results, where the perturbing body was spinless. Here, the orbit of the smaller body is still circular (or "helical" in the perturbed spacetime having a Killing helical symmetry) but nongeodesic, due to the coupling between spin and curvature as described by the MathissonPapapetrou-Dixon model for spinning bodies. Nonvanishing spin and nongeodesic orbits are then two original contributions of the present analysis to the study of twobody tidal interactions. The inclusion of spin corrections has become possible after the work of Ref. [41], where the redshift function $z_{1}$ of a spinning particle in a perturbed Schwarzschild spacetime was computed, once the completion problem of metric reconstruction was fully solved by the addition of "low multipoles" $l=0,1$.

Our results are accurate through the $9.5 \mathrm{PN}$ order for both electric and magnetic tidal invariants. We have also computed the associated eigenvalues, whose accuracy is still 9.5PN for $\lambda_{1}^{(\mathrm{E}) 1 \mathrm{SF}}$ and $\lambda^{(\mathrm{B}) 1 \mathrm{SF}}$, whereas it is one PN order less for $\lambda_{2}^{(\mathrm{E}) 1 \mathrm{SF}}$. The contribution due to spin to the $1 \mathrm{SF}$ corrections to tidal eigenvalues is in general about $0.1 \hat{s}$ times the zeroth-order-in-spin term in a spacetime region where the weak-field approximation holds. Our analytical results can be used to easily compute related gauge-invariant quantities, like the conservative part of the speciality index, whose corrections mark the change of Petrov spectral type of the (algebraically special typeD) background metric in an invariant way $37,55,56$. This information can then be useful to test numerical relativity results in the extreme-mass-ratio limit.

Another important task will be the conversion of these results into the EOB formalism. Actually, following Ref. 20] spin modification to the tidal potentials should naturally enter the EOB main radial potential $A(u)$ (see Eq. (1.1) there)

$$
A(u)=A^{\mathrm{BBH}}(u)+A_{1}^{\text {tidal }}(u)+A_{2}^{\text {tidal }}(u),
$$

where $A^{\mathrm{BBH}}(u)$ is the potential describing the dynamics of the binary system and $A_{1,2}^{\text {tidal }}(u)$ those associated with the tidal deformations of the two bodies. Spin corrections can thus be included in $A_{1,2}^{\text {tidal }}(u)$, with for instance

$$
A_{1}^{\text {tidal }}(u)=A_{1}^{\text {tidal } S_{1}^{0}}(u)+S_{1} A_{1}^{\text {tidal } S_{1}^{1}}(u)+O\left(S_{1}^{2}\right),
$$

where $S_{1}$ denotes the spin of the body 1 . However, linearin-spin terms naturally enter also the spin-orbit part of the EOB Hamiltonian, providing a tidal modification of the two gyrogravitomagnetic ratios $g_{S}$ and $g_{S^{*}}$. Indeed, the radial potential $A(u)$ incorporates typically even-inspin corrections, whereas $g_{S}$ and $g_{S^{*}}$ include odd-in-spin corrections. This problem is currently under investigation and will be addressed elsewhere.

\section{Acknowledgments}

The authors thank T. Damour for useful discussions. D.B. thanks the Naples Section of the Italian Istituto Nazionale di Fisica Nucleare (INFN) and the International Center for Relativistic Astrophysics Network (ICRANet) for partial support.
[1] B. P. Abbott et al. [LIGO Scientific and Virgo Collaborations], "Observation of Gravitational Waves from a Binary Black Hole Merger," Phys. Rev. Lett. 116, no. 6, 061102 (2016) doi:10.1103/PhysRevLett.116.061102 arXiv:1602.03837 [gr-qc]].
[2] B. P. Abbott et al. [LIGO Scientific and Virgo Collaborations], "GW151226: Observation of Gravitational Waves from a 22-Solar-Mass Binary Black Hole Coalescence," Phys. Rev. Lett. 116, no. 24, 241103 (2016) doi:10.1103/PhysRevLett.116.241103 arXiv:1606.04855 
[gr-qc]].

[3] B. P. Abbott et al. [LIGO Scientific and Virgo Collaborations], "GW170814: A Three-Detector Observation of Gravitational Waves from a Binary Black Hole Coalescence," Phys. Rev. Lett. 119, no. 14, 141101 (2017) doi:10.1103/PhysRevLett.119.141101 arXiv:1709.09660 [gr-qc]].

[4] B. P. Abbott et al. [LIGO Scientific and Virgo Collaborations], "GW170817: Observation of Gravitational Waves from a Binary Neutron Star Inspiral," Phys. Rev. Lett. 119, no. 16, 161101 (2017) doi:10.1103/PhysRevLett.119.161101 arXiv:1710.05832 [gr-qc]].

[5] E. E. Flanagan and T. Hinderer, "Constraining neutron star tidal Love numbers with gravitational wave detectors," Phys. Rev. D 77, 021502 (2008) arXiv:0709.1915 [astro-ph]].

[6] J. S. Read, C. Markakis, M. Shibata, K. Uryu, J. D. E. Creighton and J. L. Friedman, "Measuring the neutron star equation of state with gravitational wave observations," Phys. Rev. D 79, 124033 (2009) arXiv:0901.3258 [gr-qc]].

[7] L. Baiotti, T. Damour, B. Giacomazzo, A. Nagar and L. Rezzolla, "Analytic modelling of tidal effects in the relativistic inspiral of binary neutron stars," Phys. Rev. Lett. 105, 261101 (2010) arXiv:1009.0521 [gr-qc]].

[8] S. Bernuzzi, A. Nagar, M. Thierfelder and B. Brugmann, "Tidal effects in binary neutron star coalescence," Phys. Rev. D 86, 044030 (2012) arXiv:1205.3403 [gr-qc]].

[9] T. Damour, A. Nagar and L. Villain, "Measurability of the tidal polarizability of neutron stars in late-inspiral gravitational-wave signals," Phys. Rev. D 85, 123007 (2012) arXiv:1203.4352 [gr-qc]].

[10] S. Bernuzzi, M. Thierfelder and B. Bruegmann, "Accuracy of numerical relativity waveforms from binary neutron star mergers and their comparison with postNewtonian waveforms," Phys. Rev. D 85, 104030 (2012) arXiv:1109.3611 [gr-qc]].

[11] J. S. Read, L. Baiotti, J. D. E. Creighton, J. L. Friedman, B. Giacomazzo, K. Kyutoku, C. Markakis and L. Rezzolla et al., "Matter effects on binary neutron star waveforms," Phys. Rev. D 88, 044042 (2013) arXiv:1306.4065 [gr-qc]].

[12] W. Del Pozzo, T. G. F. Li, M. Agathos, C. Van Den Broeck and S. Vitale, "Demonstrating the feasibility of probing the neutron star equation of state with secondgeneration gravitational wave detectors," Phys. Rev. Lett. 111, no. 7, 071101 (2013) arXiv:1307.8338 [gr-qc]].

[13] M. Ishii, M. Shibata and Y. Mino, "Black hole tidal problem in the Fermi normal coordinates," Phys. Rev. D 71, 044017 (2005) doi:10.1103/PhysRevD.71.044017 gr-qc/0501084.

[14] T. Damour and G. Esposito-Farese, "Testing gravity to second postNewtonian order: A Field theory approach," Phys. Rev. D 53, 5541 (1996) doi:10.1103/PhysRevD.53.5541 gr-qc/9506063..

[15] W. D. Goldberger and I. Z. Rothstein, "An Effective field theory of gravity for extended objects," Phys. Rev. D 73, 104029 (2006) doi:10.1103/PhysRevD.73.104029 hep-th/0409156.

[16] R. A. Porto, "Post-Newtonian corrections to the motion of spinning bodies in NRGR," Phys. Rev. D 73, 104031 (2006) doi:10.1103/PhysRevD.73.104031 gr-qc/0511061.

[17] M. Levi and J. Steinhoff, "Leading order finite size effects with spins for inspiralling compact binaries," JHEP 1506, 059 (2015) doi:10.1007/JHEP06(2015)059 arXiv:1410.2601 [gr-qc]].

[18] M. Levi and J. Steinhoff, "Spinning gravitating objects in the effective field theory in the post-Newtonian scheme," JHEP 1509, 219 (2015) doi:10.1007/JHEP09(2015)219 arXiv:1501.04956 [gr-qc]].

[19] D. Bini and A. Geralico, "Tidal invariants along the worldline of an extended body in Kerr spacetime," Phys. Rev. D 91, no. 8, 084012 (2015). doi:10.1103/PhysRevD.91.084012

[20] D. Bini, T. Damour and G. Faye, "Effective action approach to higher-order relativistic tidal interactions in binary systems and their effective one body description," Phys. Rev. D 85, 124034 (2012) doi:10.1103/PhysRevD.85.124034 arXiv:1202.3565 [grqc]].

[21] L. Blanchet, "Gravitational Radiation from PostNewtonian Sources and Inspiralling Compact Binaries," Living Rev. Rel. 17, 2 (2014) doi:10.12942/lrr-2014-2 arXiv:1310.1528 [gr-qc]].

[22] L. Blanchet and T. Damour, "Mthode d'itration postMinkowskienne et structure des champs gravitationnels radiatifs," Compt. Rend. Acad. Sci. Ser. II 298, no. 10, 431 (1984).

[23] T. Damour, "Gravitational scattering, postMinkowskian approximation and Effective One-Body theory," Phys. Rev. D 94, no. 10, 104015 (2016) doi:10.1103/PhysRevD.94.104015 arXiv:1609.00354 [gr-qc]].

[24] S. L. Detweiler and L. H. Brown, Jr., "The PostMinkowski expansion of general relativity," Phys. Rev. D 56, 826 (1997) doi:10.1103/PhysRevD.56.826 gr-qc/9609010.

[25] L. Barack, "Gravitational self force in extreme massratio inspirals," Class. Quant. Grav. 26, 213001 (2009) doi:10.1088/0264-9381/26/21/213001 arXiv:0908.1664 [gr-qc]].

[26] K. Hotokezaka, K. Kyutoku and M. Shibata, "Exploring tidal effects of coalescing binary neutron stars in numerical relativity," Phys. Rev. D 87, no. 4, 044001 (2013) arXiv:1301.3555 [gr-qc]].

[27] D. Radice, L. Rezzolla and F. Galeazzi, "Beyond secondorder convergence in simulations of binary neutron stars in full general-relativity," Mon. Not. Roy. Astron. Soc. 437, L46 (2014) arXiv:1306.6052 [gr-qc]].

[28] S. Bernuzzi, A. Nagar, S. Balmelli, T. Dietrich and M. Ujevic, "Quasiuniversal properties of neutron star mergers," Phys. Rev. Lett. 112, 201101 (2014) arXiv:1402.6244 [gr-qc]].

[29] A. Buonanno and T. Damour, "Effective one-body approach to general relativistic two-body dynamics," Phys. Rev. D 59, 084006 (1999) gr-qc/9811091.

[30] A. Buonanno and T. Damour, "Transition from inspiral to plunge in binary black hole coalescences," Phys. Rev. D 62, 064015 (2000) gr-qc/0001013.

[31] T. Damour, P. Jaranowski and G. Schaefer, "On the determination of the last stable orbit for circular general relativistic binaries at the third postNewtonian approximation," Phys. Rev. D 62, 084011 (2000) gr-qc/0005034.

[32] T. Damour, "Coalescence of two spinning black holes: an effective one-body approach," Phys. Rev. D 64, 124013 (2001) gr-qc/0103018.

[33] A. Taracchini et al., "Effective-one-body model for black- 
hole binaries with generic mass ratios and spins," Phys. Rev. D 89, no. 6, 061502 (2014) arXiv:1311.2544 [gr-qc]].

[34] M. Pürrer, "Frequency domain reduced order model of aligned-spin effective-one-body waveforms with generic mass-ratios and spins," Phys. Rev. D 93, no. 6, 064041 (2016) arXiv:1512.02248 [gr-qc]].

[35] J. Steinhoff, T. Hinderer, A. Buonanno and A. Taracchini, "Dynamical Tides in General Relativity: Effective Action and Effective-One-Body Hamiltonian," Phys. Rev. D 94, no. 10, 104028 (2016) doi:10.1103/PhysRevD.94.104028 arXiv:1608.01907 [gr-qc]].

[36] D. Bini and T. Damour, "Gravitational self-force corrections to two-body tidal interactions and the effective onebody formalism," Phys. Rev. D 90, no. 12, 124037 (2014) doi:10.1103/PhysRevD.90.124037 arXiv:1409.6933 [grqc]].

[37] S. R. Dolan, P. Nolan, A. C. Ottewill, N. Warburton and B. Wardell, "Tidal invariants for compact binaries on quasicircular orbits," Phys. Rev. D 91, no. 2, 023009 (2015) doi:10.1103/PhysRevD.91.023009 arXiv:1406.4890 [gr-qc]].

[38] C. Kavanagh, A. C. Ottewill and B. Wardell, "Analytical high-order post-Newtonian expansions for extreme mass ratio binaries," Phys. Rev. D 92, no. 8, 084025 (2015) doi:10.1103/PhysRevD.92.084025 arXiv:1503.02334 [grqc]].

[39] P. Nolan, C. Kavanagh, S. R. Dolan, A. C. Ottewill, N. Warburton and B. Wardell, "Octupolar invariants for compact binaries on quasicircular orbits," Phys. Rev. D 92, no. 12, 123008 (2015) doi:10.1103/PhysRevD.92.123008 arXiv:1505.04447 [grqc]].

[40] A. G. Shah and A. Pound, "Linear-in-mass-ratio contribution to spin precession and tidal invariants in Schwarzschild spacetime at very high post-Newtonian order," Phys. Rev. D 91, no. 12, 124022 (2015) doi:10.1103/PhysRevD.91.124022 arXiv:1503.02414 [grqc]].

[41] D. Bini, T. Damour, A. Geralico and C. Kavanagh, "Detweilers redshift invariant for spinning particles along circular orbits on a Schwarzschild background," Phys. Rev. D 97, no. 10, 104022 (2018) doi:10.1103/PhysRevD.97.104022 arXiv:1801.09616 [grqc]].

[42] M. Mathisson, "Neue mechanik materieller systemes," Acta Phys. Polon. 6, 163 (1937).

[43] A. Papapetrou, "Spinning test particles in general relativity. 1.," Proc. Roy. Soc. Lond. A 209, 248 (1951). doi:10.1098/rspa.1951.0200

[44] W. G. Dixon, "Dynamics of extended bodies in general relativity. I. Momentum and angular momentum," Proc. Roy. Soc. Lond. A 314, 499 (1970). doi: $10.1098 /$ rspa. 1970.0020

[45] W. Tulczyjew, "Motion of multipole particles in general relativity theory," Acta Phys. Polon. 18, 393 (1959).

[46] D. Bini and T. Damour, "Two-body gravitational spin-orbit interaction at linear order in the mass ratio," Phys. Rev. D 90, no. 2, 024039 (2014) doi:10.1103/PhysRevD.90.024039 arXiv:1404.2747 [gr$\mathrm{qc}]]$.

[47] L. Blanchet, A. Buonanno and A. Le Tiec, "First law of mechanics for black hole binaries with spins," Phys. Rev. D 87, no. 2, 024030 (2013) doi:10.1103/PhysRevD.87.024030 arXiv:1211.1060 [grqc]].

[48] S. R. Dolan, N. Warburton, A. I. Harte, A. Le Tiec, B. Wardell and L. Barack, "Gravitational self-torque and spin precession in compact binaries," Phys. Rev. D 89, no. 6, 064011 (2014) doi:10.1103/PhysRevD.89.064011 arXiv:1312.0775 [gr-qc]].

[49] T. Regge and J. A. Wheeler, "Stability of a Schwarzschild singularity," Phys. Rev. 108, 1063 (1957). doi:10.1103/PhysRev.108.1063

[50] F. J. Zerilli, "Gravitational field of a particle falling in a schwarzschild geometry analyzed in tensor harmonics," Phys. Rev. D 2, 2141 (1970). doi:10.1103/PhysRevD.2.2141

[51] S. Mano, H. Suzuki and E. Takasugi, "Analytic solutions of the Regge-Wheeler equation and the postMinkowskian expansion," Prog. Theor. Phys. 96, 549 (1996) gr-qc/9605057.

[52] S. Mano, H. Suzuki and E. Takasugi, "Analytic solutions of the Teukolsky equation and their low frequency expansions," Prog. Theor. Phys. 95, 1079 (1996) gr-qc/9603020.

[53] D. Bini and T. Damour, "Analytical determination of the two-body gravitational interaction potential at the fourth post-Newtonian approximation," Phys. Rev. D 87, no. 12, 121501 (2013) doi:10.1103/PhysRevD.87.121501 arXiv:1305.4884 [gr-qc]].

[54] A. Heffernan, A. Ottewill and B. Wardell, "Highorder expansions of the Detweiler-Whiting singular field in Schwarzschild spacetime," Phys. Rev. D 86, 104023 (2012) doi:10.1103/PhysRevD.86.104023 arXiv:1204.0794 [gr-qc]].

[55] C. Cherubini, D. Bini, M. Bruni and Z. Perjes, "Petrov classification of perturbed space-times: The Kasner example," Class. Quant. Grav. 21, 4833 (2004) doi:10.1088/0264-9381/21/21/006 gr-qc/0404075.

[56] C. Cherubini, D. Bini, M. Bruni and Z. Perjes, "The Speciality index as invariant indicator in the BKL mixmaster dynamics," Class. Quant. Grav. 22, 1763 (2005) doi:10.1088/0264-9381/22/9/018 [gr-qc/0408040]. 